\title{
CORRIGENDUM
}

to

\section{"RICE PLANT DISEASE CLASSIFICATION USING TRANSFER LEARNING OF DEEP CONVOLUTION NEURAL NETWORK"}

by V. K. Shrivastava, M. K. Pradhan, S. Minz, and M. P. Thakur,

Int. Arch. Photogramm. Remote Sens. Spatial Inf. Sci., XLII-3/W6, 631-635, 2019.

The following paper has to be considered as a replacement of the originally published version. The paper was sent by mistake in an incorrect version to the Copernicus Service Provider. Corrected section: 1 Introduction.

The Editors apologize with the Authors for the inconvenience.

17 August 2019 


\title{
RICE PLANT DISEASE CLASSIFICATION USING TRANSFER LEARNING OF DEEP CONVOLUTION NEURAL NETWORK
}

\author{
Vimal K. Shrivastava ${ }^{1}$, Monoj K. Pradhan ${ }^{2 *}$, Sonajharia Minz ${ }^{3}$, Mahesh P. Thakur ${ }^{4}$ \\ ${ }^{1}$ School of Electronics Engineering, KIIT, Bhubaneswar, India- vimal.shrivastavafet@kiit.ac.in \\ ${ }^{2}$ Department of Agricultural Statistics \& Computer Science, IGKV, Raipur, India- monojpradhan76@gmail.com, \\ ${ }^{3}$ School of Computer \& Systems Sciences, JNU, New Delhi, India- Sona.minz@gmail.com \\ ${ }^{4}$ Department of Plant Pathology, IGKV, Raipur, India- mp_thakur@yahoo.com
}

Commission VI, WG VI/4

KEY WORDS: Classification, CNN, Deep Learning, Pattern Recognition, Rice Plant Disease, Transfer Learning.

\begin{abstract}
:
Early and accurate diagnosis of plant diseases is a vital step in the crop protection system. In traditional practices, identification is performed either by visual observation or by testing in laboratory. The visual observation requires expertise and it may vary subject to an individual which may lead to an error while the laboratory test is time consuming and may not be able to provide the results in time. To overcome these issues, image based machine learning approach to detect and classify plant diseases has been presented in literature. We have focused specifically on rice plant (Oryza Sativa) disease in this paper. The images of the diseased symptoms in leaves and stems have been captured from the rice field. We have collected a total of 619 rice plant diseased images from the real field condition belong to four classes:(a) Rice Blast (RB), (b) Bacterial Leaf Blight (BLB), (c) Sheat Blight (SB) and (d) Healthy Leave (HL).We have used a pre-trained deep convolutional neural network(CNN) as a feature extractor and Support Vector Machine (SVM) as a classifier. We have obtained encouraging results. The early identification of rice diseases by this approach could be used as a preventive measure well as an early warning system. Further, it could be extended to develop a rice plant disease identification system on real agriculture field.
\end{abstract}

\section{Introduction}

Crop plant disease identification and classification, in the early stage is one of the most vital activities in agricultural practices. Infection by diseases results in great loss to economic to the farmers in every year. Therefore, quickly, timely and accurate diagnosis of the disease prevents the loss of product and improves the quality of the product as well (Phadikar et al., 2013). As a consequence, it helps in economic growth of the country. In traditional practices, identification of these diseases are based either on the visual symptoms caused by the pathogens or identification of pathogens in the laboratory (Barbedo, 2016). The visual assessment of the disease lesion is that it is subjective matter and may fail to diagnosis the disease successfully. On the contrary, pathogen identification in laboratory becomes a cumbersome process as it is time taking process for pathogen culturing which may fail to provide results in appropriate time (Barbedo, 2016). Moreover, both of the above mentioned procedures require professionals along with rich experienced experts to identify the diseases. These limitations have encouraged the researchers to explore automatic methods that detect and classify plant diseases in a simple and reliable way with high accuracy. In addition, it will help the farmers to select the correct pesticides. Along this line, image based plant disease identification and classification models have been developed in literature for various plants such as (Liu et al., 2009; Phadikar et al.,2013; Joshi et al., 2016; Jia et al., 2013; Hu et al., 2016; Cao et al., 2012). Among different crops, rice is the major crop in Asian country (Liu et al., 2010) including India (Shah et al., 2016). In this paper, we have focused specifically on rice plant disease identification and classification.

According to statistics, $10-15 \%$ of rice production in Asia is destroyed because of rice plant disease (Gianessi et al., 2014). The fungus and bacteria are believed to be the main reason for rice plant diseases. There are various rice plant diseases. In this paper, we have considered the images of three common rice plant diseases: Rice Blast (RB), Bacterial Leaf Blight (BLB) and Sheath Blight (SB) along with the healthy (HL) images.

Rice plant disease identification and classification are dependent on the symptoms and signs produced by the pathogens. Many a time, the identification of disease on the basis of symptoms becomes very difficult. Therefore, identification based on digital images has been increasingly growing (Barbedo, 2013). Multispectral and hyperspectral images provide more information, but they are more expensive for farmers. While the conventional cameras and in-built cameras in mobile phones are easily available with almost all farmers with affordable cost to capture images. This has prompted the researchers in developing systems based on RGB color images i.e. in the visible range. Hence, we have used RGB color images in this study as well.

In this new era of science and technology, many computeraided diagnosis (CAD) systems has been developed for agricultural sciences along with plant disease recognition CAD systems (Hu et al., 2016; Baldi et al., 2017; Chaki et al., 2015). Among different methods, traditional machine learning techniques is one; which have been used in literature for rice plant disease classification. In traditional approaches, the characteristic features from the diseased images are extracted by applying various image processing techniques and then different classification techniques such as discriminant analysis, decision tree, neural network and support vector machine are applied for disease recognition.

Suresha et al., 2017, applied the global thresh holding methods to extract disease part of rice leaves. Further, the authors used

* Corresponding author 
k-nearest neighbour ( $\mathrm{k}-\mathrm{NN})$ classifier to recognize rice blast and brown spot. Prajapati et al., 2017, proposed centroid feeding based K-means clustering for segmentation and further removed the ineffective spots and used SVM to classify the rice disease. Sanyal et al., 2008, proposed the method of extracting features of the rice diseases i.e. color and texture and these features were fed to multilayer perceptron (MLP) to detect different rice plant diseases. Joshi et al., 2016, extracted the color and shape features of the leaf and used these features to minimum distance classifier (MDC) and $\mathrm{k}-\mathrm{NN}$ Classifier to detect the diseases. In the same line, Yao et al., 2009, proposed the combination of texture and shape features of the rice leaf to detect the different rice diseases using the SVM classifier. Majid et al., 2013, used fuzzy entropy and probabilistic neural network classifier to identify diseases. Xiao et al., 2018 explored principal component analysis (PCA) for dimensionality reduction of features and used back propagation (BP) neural network model to classify the different rice diseases.

Now days, deep learning techniques have attracted the attention of researchers due to its great performance in image classification. The advantage of deep learning technique is that it avoids extraction of complex hand-crafted features unlike traditional machine learning techniques and provides end-toend learning. Among different deep learning techniques, the deep convolutional neural network (CNN) has been used mostly for image classification (Krizhevsky et al., 2012; Lu et al., 2017). The CNN model provides a relationship between layers and spatial information of the image and hence it is convenient for the classification of image (Arel et al., 2010). Along this line, there are limited works on rice plant disease classification using CNN. Lu et al., 2017, investigated the ability of deep $\mathrm{CNN}$ technique for classification of different rice diseases. A total of 500 images belonging to 10 categories have been considered and used CNN model with three convolution layers, three stochastic pooling layers and softmax layer at the end. The classification accuracy of $95.48 \%$ has been reported. Rajmohan et al., 2018 have explored the deep CNN for denoising images and used SVM for rice disease classification using shape and color features. A total of 200 images belonging to seven categories have been considered. They reported a classification accuracy of $87.50 \%$. However, CNN requires massive training data for proper training and collection of rice diseased images is a challenging task. This limitation can be overcome by the application of transfer learning. The transfer learning technique uses previously trained networks on large dataset to update the weights for current small dataset (Bengio, 2012). In this paper, we have used AlexNet (Krizhevsky et al., 2012) which is a deep CNN model pre-trained on large ImageNet dataset (Deng et al., 2009). To the best of our knowledge, this is the first paper which explores the application of transfer learning technique for rice plant disease classification.

\section{Methodology}

In recent times, the deep learning techniques have obtained very high performance in almost all aspects of the problems, such as image recognition (Litjens et al., 2017), image segmentation (Long et al., 2015; Garcia et al., 2017), speech recognition (Abdel-Hamid et al., 2014), natural language processing (Young et al., 2018), emotion recognition (Mohammadpour et al., 2017), recommendation system (Zhang et al., 2017) etc. In general, deep learning technique is end-to-end learning and thus avoids complex hand-crafted feature extraction. It learns features at different levels of abstraction as layer increases. The deep convolutional neural network (CNN) is the most popular and extensively used for image recognition (Lu et al., 2017). It mainly comprises of convolutional, pooling and fully-connected layers. However, CNN requires large labelled dataset such as ImageNet (Denget al., 2009) to train efficiently which is a challenging task in the field of agriculture. Otherwise, the performance of $\mathrm{CNN}$ is not encouraging on small datasets because of over fitting (Hnoohom et al., 2018). Transfer learning is a technique, where the initialization of $\mathrm{CNN}$ weights occurs from pre-trained network(Oquab et al., 2014; Hu et al., 2015; Ng et al., 2015;Hoo-Chang et al., 2016). It has been observed that the performance of transfer learning is better as compared to training the network from scratch on the small dataset. We have used AlexNet (Krizhevsky et al., 2012) which is a deep CNN model pre-trained on large ImageNet dataset (Deng et. al, 2009). ImageNet dataset contains 1.2 million images of 1000 classes and AlexNet consists of five convolutional layers, three pooling layers and two fullyconnected layers as shown in Figure 2. The first layer of any CNN model defines the input dimensions. The AlexNet CNN model used in this paper requires input dimension to be $227 \times 227 \times 3$. Hence, all the input images of rice plant diseases have been resized to this dimension. The final layer of any CNN model is the classification layer. However, we have used AlexNet CNN model as a feature extractor here and not for classification task. Therefore, we have removed the last layer of AlexNet model. The extracted image features from previous deep layers are fed to Support Vector Machine (SVM) (Wang et al., 2005) classifier for training.

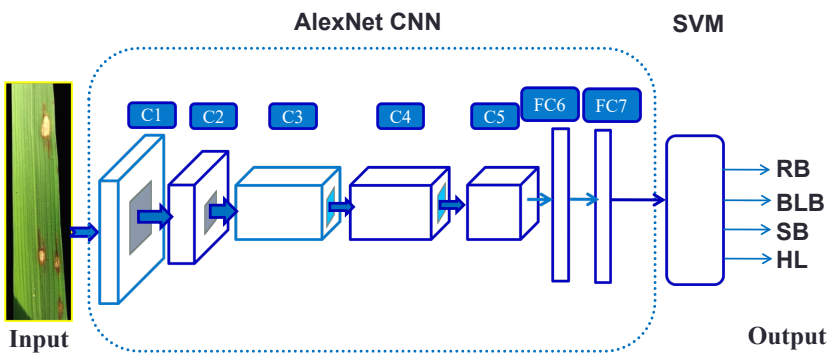

Figure 2. AlexNet Architecture

\section{Results and Discussion}

\subsection{Data Description}

The image samples used in this paper were collected from rice field of Indira Gandhi Agricultural University, Raipur, Chhattisgarh, India. These images were collected between $6.30 \mathrm{am}$ to $9.30 \mathrm{am}$ and $4.00 \mathrm{pm}$ to $5.30 \mathrm{pm}$. The image acquired during this period gives more clarity than other sunny time of the day. The images are acquired using Canon Powershot SX530HS digital camera, Gionee and LYF mobile set. The image background is prepared to be black to reduce the background complexity as a result to reduce the computation cost. There are 619 images belong to four classes: (i) Rice Blast (RB); (ii) Bacterial Leaf Blight (BLB); (ii) Sheath Blight (SB); and (iv) Healthy Leaves (HL).This is the first time when such a large rice disease dataset has been collected from Indian agriculture field.Table 1 shows the description of considered rice diseases with sample images of each class and the details are given below: 


\section{A. Rice Blast (RB)}

RB disease is incited by Magnaporthe oryzae (Couch et al., 2002). The symptoms of this disease are grayish green circular and a spot with dark green border in the leaves (Padhikar et al., 2013). The lesions are elliptical or spindle shaped. It is one of the most devastating diseases of rice in different countries of the world (Kumar et al., 2016).A total of 88 images of RB disease has been collected for this study. An example of infected leaf with RB disease is shown in Table 1.

\section{B. Bacterial Leaf Blight (BLB)}

BLB is incited by Xanthomonas oryzae. The symptoms of this disease contain a lesion that generally starts from the tip of the leaf and spread towards the base of the leaf with several inches (Rice Doctor, 2003; OU, 1985). BLB is one of the destructive diseases that affect considerable loss in the rice production (Patil et al., 2017). A total of 240 images of BLB disease have been collected for this study. An example of infected leaf with BLB disease is shown in Table 1 .

\section{Sheath Blight (SB)}

SB disease is incited Rhizoctonia solani (Nandakumar et al., 2001). The symptoms of this disease start from lower sheath of the plant and then spread towards the upper sheath and leaves. It communicates from plant to plant in the field (Zhou et al., 2015). SB has become the most economically destructive disease in major rice growing countries in the world (Zhou et al., 2015).A total of 100 images of SB disease has been collected for this study. An example of infected leaf with SB disease is shown in Table 1.

\begin{tabular}{|c|c|c|}
\hline $\begin{array}{c}\text { Rice Disease } \\
\text { Class Name }\end{array}$ & $\begin{array}{c}\text { Total Number of } \\
\text { Images }\end{array}$ & Sample Image \\
\hline $\mathrm{RB}$ & 88 & \\
\hline $\mathrm{BLB}$ & 240 & \\
\hline $\mathrm{SB}$ & 100 & \\
\hline $\mathrm{HL}$ & 191 & \\
\hline
\end{tabular}

Table 1. Details of Four Rice Diseases in the Dataset with Sample Images from Each Class

\subsection{Experiment Setup and Performance Analysis}

This paper investigates the use of transfer learning of deep $\mathrm{CNN}$ for rice disease classification. We have used AlexNet for extraction of features and SVM for classification. The MatConvNet toolbox (Vedaldi et al.) is used for implementation of AlexNet CNN model.The considered dataset has been randomly partitioned into training and testing set. To analysis the effect of partitioning, three sets of training-testing partition has been done: (i) $60 \%-40 \%$; (ii) $70 \%-30 \%$; and (iii) $80 \%-20 \%$. The experiment was carried out for 10 trails $(10$ times) by choosing samples randomly for each partition from whole dataset. Further, the classification accuracy was calculated by averaging the accuracy for 10 trails. The results are shown in Table 2. All of our experiments were performed on Intel Core i5-6200U CPU @2.40 GHz with 8GB RAM and NVIDIA GeForce 940M GPU. The classification accuracy of $91.37 \%$ has been achieved with training-testing partition of $80 \%-20 \%$.

\begin{tabular}{|c|c|}
\hline $\begin{array}{c}\text { Training-Testing } \\
\text { Division (\%) }\end{array}$ & $\begin{array}{c}\text { Classification } \\
\text { Accuracy (\%) }\end{array}$ \\
\hline $60-40$ & 89.45 \\
$70-30$ & 90.39 \\
$80-20$ & 91.37 \\
\hline
\end{tabular}

Table 2. Classification Accuracy with Different TrainingTesting Partition.

\section{Conclusion}

In the Indian scenario, a limited works have been done on automatic classification of rice plant diseases. In this paper, transfer learning of deep $\mathrm{CNN}$ was explored first time to classify rice plant diseases. Moreover, the experiments were conducted by partitioning the whole dataset into different ratio of training-testing set. The proposed model is able to classify rice diseases with classification accuracy of $91.37 \%$ for $80 \%$ $20 \%$ training-testing partition. However, benchmarking of proposed model with literature is not appropriate because of unavailability of standard labelled rice disease images. The performance of proposed model can be further improved with large dataset of rice diseased images.

\section{References}

Abdel-Hamid, O., Mohamed, A., Jiang, H., Deng, L., Penn, G., \& Yu, D.(2014) Convolutional neural networks for speech recognition. IEEE/ACM Transactions on Audio, Speech, and Languge processing, 22 (10), 1533-1545.

Bengio, Y. (2012). Deep Learning of Representations for Unsupervised and Transfer Learning. Proceedings of ICML Workshop on Unsupervised and Transfer Learning, PMLR, 27, pp. 17-36.

Cao, L.Y., Zhang, X.X., San, X.H., \& Chen, G.F.(2012). Research on the method of diagnosis for maize disease based on image processing technology and BP neural network algorithm,Comput. Sci., 39 (10).

Couch, B.C., \& Kohn, L.M.(2002). A multilocus gene genealogy concordant with host preference indicates segregation of new species, Magnaporthe oryzae, from M. grisea, Micrologica, 94(4), 683-693.

Barbedo, J. G. A. (2016). A review on the main challenges in automatic plant disease identification based on visible range images. Bio systems Engineering, 144, 52-60.

Barbedo, J. G. A. (2013).Digital image processing techniques for detecting, quantifying and classifying plant diseases. SpringerPlus, 2:660, 1-12

Deng, J., Dong, W., Socher, R., Li, L. J., Li, K., \& Fei-Fei, L. (2009). Imagenet: A large-scale hierarchical image database. In Computer Vision and Pattern Recognition, 248-255. 
Garcia-Garcia, A., Orts-Escolano, S., Oprea, S., VillenaMartinez, V., \& Garcia-Rodriguez, J. (2017). A review on deep learning techniques applied to semantic segmentation. arXiv preprint arXiv:1704.06857.

Gianessi, L. P. (2014). Importance of pesticides for growing rice in South and South East Asia. Crop Life Foundation, 3033.

Hnoohom, N., \& Yuenyong, S. (2018, July). Classification of dhamma esan characters by transfer learning of a deep neural network. In 2018 15th International Joint Conference on Computer Science and Software Engineering (JCSSE) (pp. 1-5). IEEE.

Hoo-Chang, S., Roth, H. R., Gao, M., Lu, L., Xu, Z., Nogues, I., \& Summers, R. M. (2016). Deep convolutional neural networks for computer-aided identification: $\mathrm{CNN}$ architectures, dataset characteristics and transfer learning. IEEE Transactions on Medical Imaging, 35(5), 1285-1298.

Hu, F., Xia, G. S., Hu, J., \& Zhang, L. (2015). Transferring deep covolutional neural networks for the scene classification of high resolution remote sensing imagery. Remote Sensing, 7(11), 14680-14707.

Hu, Y.H., Ping, X.W., Xu, M.Z., Dan, W.X.,\& He, Y.(2016). Detection of late blight disease on potato leaves using hyperspectral imaging technique. Spectrosc. Spect. Anal. 36 (2), 515-519.

Jia, J.N.,\& Ji, H.Y. (2013). Recognition for cucumber disease based on leaf spot shape and neural network. Trans. Chinese Soc. Agric. Eng. 29 (S1), 115-121.

Joshi A. A., \& Jadhav B.D. (2016). Monitoring and controlling rice diseases using image processing techniques. International Conference on Computing, Analytics and Security Trends (CAST), pp. 471-476.

Krizhevsky, A., Sutskever, I., \& Hinton, G. E. (2012). Imagenet classification with deep convolutional neural networks In Advances in Neural Information Processing Systems (pp. 1097-1105).

Kumar, A., Mishra, A.K., \& Jain, A.K. (2016). In-silico identification of inhibitors for controlling rice blast. International Conference on Computing for Sustainable Global Development (INDIACom), pp. 1888-1892.

Litjens, G., Kooi, T., Bejnordi, B. E., Setio, A. A. A., Ciompi, F., Ghafoorian, M.,\& Sánchez, C. I. (2017). A survey on deep learning in medical image analysis. Medical Image Analysis, 42, 60-88.

Liu, L.B., \& Zhou, G.M., (2009). Identification method of rice leaf blast using multilayer perception neural network. Transactions of the Chinese Society of Agricultural Engineering, 25 (S2).

Liu Z. Y., Wu , H. F., \& Huang, J. F.(2010) Application of neural networks to discriminate fungal infection levels in ricepanicles using hyperspectral reflectance and principal components analysis. Computers and Electronics in Agriculture, 72 , 99-106.
Long, J., Shelhamer, E., \& Darrell, T. (2015). Fully convolutional networks for semantic segmentation. In Proceedings of the IEEE Conference on Computer Vision and Pattern Recognition (pp. 3431-3440).

Lu, Y., Yi, S., Zeng, N., Liu, Y., \&Zhang,Y.(2017). Identification of rice diseases using deep convolutional neural networks. Neurocomputing, 267, 378-384.

Mohammadpour, M., Khaliliardali,H., Hashemi , S. M. R., \& AlyanNezhadi, M. M .(2017). Facial emotion recognition using deep convolutional networks. IEEE 4th International Conference on Knowledge-Based Engineering and Innovation (KBEI), Tehran, Iran, pp. 17-21.

Majid, K., Herdiyeni, Y., \& Rauf, A.(2013). I-PEDIA: Mobile Application for Paddy Disease Identification using Fuzzy Entropy and Probabilistic Neural Network. ICACSIS, pp-403406.

Nandakumar, R., Babu, S., Viswanathan, R., Raguchander, T., \& Samiyappan, R. (2001). Induction of systemic resistance in riceagainst sheath blight disease by Pseudomonas fluorescens. Soil Biol. Biochem, 33, 603-612.

Ng, H. W., Nguyen, V. D., Vonikakis, V., \& Winkler, S. (2015, November). Deep learning for emotion recognition on small datasets using transfer learning. In Proceedings of the 2015 ACM on International Conference on Multimodal Interaction, pp. 443-449.

Oquab, M., Bottou, L., Laptev, I., \& Sivic, J. (2014). Learning and transferring mid-level image representations using convolutional neural networks. In Proceedings of the IEEE Conference on Computer Vision and Pattern Recognition,pp. 1717-1724

Ou, S.H., 1985. Rice Diseases. Commonwealth Mycological Institute, England

Patil, B., Jagadeesh G. B. ,Karegowda C., Naik, S., \& Revathi R. M.(2017). Management of bacterial leaf blight of rice caused by Xanthomonas oryzaepv. oryzae under field condition. Journal of Pharmacognosy and Phytochemistry, 6(6): 244-246.

Phadikar, S., Sil,J., \& Das, A. K.,(2013). Rice diseases classification using feature selection and rule generation techniques. Computers and Electronics in Agriculture, 90, 7685 .

Prajapati „H. B., Shah J. P. \& Dabhi V. K.(2017).Detection and classification of rice plant diseases. Intelligent Decision Technologies. pp. 1-29.

Rajmohan, R., Pajany, M., Rajesh, R., Raman, D.R., \& Prabu, U. (2018). Smart paddy crop disease identification and management using deep convolution neural network and SVM classifier. International Journal of Pure and Applied Mathematics,118(15), 255-264.

Rice Doctor, 2003. International Rice Research Institute, Philipines. http://www.irri.org

Sanyal, P., \& Patel, S. C.(2008). Pattern recognition method to detect two diseases in rice plants. The Imaging Science Journal, $56,319-325$. 
Suresha, M., Shreekanth, K. N. \& Thirumalesh, B. V.(2017). Recognition of diseases in paddy leaves using kNN classifier. $2^{\text {nd }}$ International Conference for Convergence in Technology (I2CT), pp. 663-666.

Shah,J. P. , Prajapati,H. B. , \& Dabhi ,V. K.(2016) A Survey on detection and classification of rice plant diseases. IEEE International Conference on Current Trends in Advanced Computing (ICCTAC), Bangalore, India.

Wang, L. (Ed.). (2005). Support vector machines: theory and applications, Springer Science \& Business Media (Vol. 177).

Xiao, M., Ma, Y., Feng, Z., Deng, Z., Hou, S., Shu, L., \& Lu, Z.(2018) Rice blast recognition based on principal component analysis and neural Network. Computers and Electronics in Agriculture, 154, 482-490.

Yao, Q., Guan ,Z., Zhou , Y., Tang, J., Hu ,Y., \& Yang, B.(2009). Application of support vector machine for detecting rice diseases using shape and color texture features.

Young, T., Hazarika, D., Poria, S., \& Cambria, E. (2018). Recent trends in deep learning based natural language processing. IEEE Computational Intelligence Magazine, 13(3), $55-75$.

Zhang, S., Yao, L., \& Sun, A. (2017). Deep learning based recommender system: A survey and new perspectives. arXiv preprint arXiv:1707.07435.

Zhou X. G., \& Jo, Y. K.(2015). Disease management. The Texas Rice Production Guidelines. Texas AgriLife Research and Texas AgriLife Extension. B-6131, pp. 44-56.

Arel, I., Rose, C., and Karnowski, T.(2010). Deep Machine Learning - A New Frontier in Artificial Intelligence. IEEE Computational Intelligence Magazine. 5(4), 13-18. 